NASA Technical Memorandum 87321

\title{
Temperature Effects on the Universal Equation of State of Solids
}

(NASA-TM-87321) TEMPEFATURE EFFECTS ON THE UNIVERSAL EQUATION OF STATE OF SCIIDS (NASA) $29 \mathrm{p} \mathrm{HC} \mathrm{A03/MF} \mathrm{AO1}$ CSCL 08M
N $86-28776$

Unclas

G3/77 43383

Pascal Vinet and John Ferrante

Lewis Research Center

Cleveland, Ohio

and

John R. Smith

General Motors Research Laboratories

Warren, Michigan

and

James H. Rose

Ames Laboratory-USDOE

Ames, lowa

April 1986

NASA 
TEMPERATURE EFFECTS ON THE UNIVERSAL EQUATION OF STATE OF SOLIDS

\author{
Pascal Vinet* and John Ferrante \\ National Aeronautics and Space Administration \\ Lewis Research Center \\ Cleveland, Ohio 44135
}

and

John R. Smith

General Motors Research Laboratories

Warren, Michigan 48090

and

James H. Rose

Ames Laboratory - USOOE

Iowa State University

Ames, Iowa 50011

\title{
ABSTRACT
}

Recently it has been argued based on theoretical calculations and experimental data that there is a universal form for the equation of state of solids. This observation was restricted to the range of temperatures and pressures such that there are no phase transitions. The use of this universal relation to estimate pressure-volume relations (i.e., isotherms) required three input parameters at each fixed temperature. In this paper we show that for many solids the input data needed to predict high temperature thermodynamical properties can be dramatically reduced. In particular, only four numbers are needed: (1) the zero pressure $(P=0)$ isothermal bulk modulus; (2) its $P=0$ pressure derivative; (3) the $P=0$ volume; and (4) the $P=0$ thermal expansion; all evaluated at a single (reference) temperature. Explicit predictions are made for the high temperature isotherms, the thermal expansion as a function of temperature, and the temperature variation of the

*Visiting.scientist from Laboratoire de Technologie des Surfaces, Ecole Centrale de Lyon, B.P. 163, 69131 Ecully Cedex, France. 
isothermal bulk modulus and its pressure derivative. These predictions are tested using experimental data for three representative solids: gold, sodium chloride and xenon. Good agreement between theory and experiment is found.

\section{INTRODUCTION}

Knowledge of the equation of state (the pressure, volume, temperature $(P-V-T)$ relation), based on either calculation or measurement, is of primary importance in both basic and applied sciences. It provides insight into the nature of solid state theories, and determines the values of fundamental thermodynamic parameters.

Many theoretical and semi-empirical calculations have been carried out to describe the isothermal equation of state (EOS). Analytic semi-empirical expressions for the EOS for each class of solid abound in a variety of forms, (Ref. 1), with care often taken to present different forms for different classes of solids (Refs. 1 to 3 ). We have found, however, that there is a universal EOS for all classes of solids in compression and in the absence of phase transitions (Ref. 4). Further, we found that this universal EOS can be predicted from a knowledge of three equilibrium quantities at each fixed temperature $T$ : the equilibrium volume $V_{0}$, isothermal bulk modulus $B_{0}$, and isothermal $(\partial B / \partial P)_{0}$. Here, we use the notation $(\partial B / \partial P)_{0}$ for the zero pressure value of the isothermal pressure derivative of the isothermal bulk modulus: $(\partial B / \partial P)_{T, P}=0^{\circ}$

In the present study we will show how to predict high temperature properties of the EOS based on an even smaller amount of input data: the thermal expansion coefficient $\alpha_{0}, V_{0}, B_{0}$ and $(\partial B / \partial P)_{0}$ at zero pressure and at a single (reference) temperature. This approach, involving input data at a single temperature, applies to solids whose thermal pressure is essentially independent of the volume and linear with temperature above the Debye temperature. This property of near volume independence and linearity 
with temperature has been found for a number of solids (see, e.g., Refs. 5 to 8). Based on this assumption the new approach not only predicts the pressure-volume relation at high temperatures, but also the temperature dependence of the thermal expansion, $B_{0}$, and $(\partial B / \partial P)_{0}$. We will see that rather good accuracy can be obtained with these predictions in comparison with experimental data for some typical solids (a metal, an alkali-halide and a rare-gas solid). Even nonlinear effects in the thermal expansion, which have been difficult to obtain (Ref.9), are accurately predicted in this approach. In the following we will first briefly review the observation of a universal EOS for all classes of solids in compression (Ref. 4). Next we will formulate a method to predict high temperature properties of the universal EOS based only on zero pressure data at a reférence temperature. We then present a simple analytical approximation to the universal EOS which is then used as the reference isotherm. The last section will show a set of predictions for the thermodynamic properties of some typical solids (a metal, an alkali-halide crystal, and a rare-gas solid). These predictions will include high temperature pressure-volume curves, thermal expansion curves, and the temperature dependence of the isothermal bulk modulus and its pressure derivative.

\section{A UNIVERSAL EQUATION OF STATE}

A universal energy relation between the total energy $E$ and $a$ characteristic distance has been discovered for bimetallic adhesion (Ref. 10), chemisorption on metals (Ref. 11) and metallic cohesion (Ref. 12) (and even then hypothesized for the EOS of nuclear matter (Ref. 13)). This relation can be written as

$$
E(a)=\Delta E E^{*}\left(a^{*}\right)
$$


where

$$
a^{*}=\frac{\left(r_{W S}-r_{W S e}\right)}{\ell}
$$

Here $\Delta E$ is' the equilibrium binding energy for metallic cohesion, $r_{W S}$ is the wigner-Settz radius with $P=0$ value of $r_{\text {wSe }}$ and $\ell$ is a scaling length.

Consider now the classes of solids other than metals. There is some evidence (Refs. 12 and 14) that covalent bonds satisfy Eq. (2.1). However, rare gas and ionic bonds do not. For energy relations in either compression or expansion, ionic and rare-gas interactions are of inherently different form than the wave-function-overlap interactions (Ref. 15) characteristic of metallic and covalent bonds. It is these differences that have led to the proposal of different forms for the EOS of different classes of solids (Refs. 1 and 2).

However we have found that in compression the form of the pressure-volume relation is dominated by overlap interactions for all classes of solids. That is, at $T=0 \mathrm{~K}$, the pressure $P(V)$ has the form

$$
P(V)=-d E / d V
$$

for all classes of solids, where $E$ ts the universal form for the energy (Eq. (2.1)) and $v=4 \pi r_{W S}^{3} / 3$ is the volume. Thus many different forms are not needed to describe the EOS. Rather, a single universal expression suffices.

In this paper we are primarily interested in temperature effects. For $T>0$ Eq. (2.3) becomes

$$
P(T, V)=-(\partial F(T, V) / \partial V)_{T}=-d E(V) / d V+P *(T, V)
$$

where $F(T, V)$ is the Helmholtz free energy, which includes a temperature dependent internal energy and entropy, and $P^{\star}(T, V)$ is the thermal pressure. Now by definition, $P \star(T, V) \rightarrow 0$ as $T \rightarrow 0$. Thus for low $T,-d E / d V$ 
dominates and one might expect the form of $P(T, V)$ to be similar to that of - $d E(V) / d V$ with the temperature dependence being contained in the scaling parameters. For higher temperatures, $P^{*}(T, V)$ is not negligible, but in general it is known (Refs. 5 to 8,16 , and 17 ) to be a weak function of $v$. In this case," again one might expect that the form of - $d E(V) / d V$ and $P(T, V)$ would be similar. with the scaling parameters containing the temperature information.

To make this more concrete, we first define the function. $H$ as

$$
H=\frac{\left(\frac{V}{V_{0}}\right)^{2 / 3}}{3\left[1-\left(\frac{V}{V_{0}}\right)\right]^{1 / 3}} P
$$

This definition is prompted by the form of - $d E(V) / d V$ obtained from the universal energy relation, Eq. (2.1), for metals (Ref. 18). Our results for metals (Ref. 18) suggest that plots of $\ln H$ versus $1-\left(\mathrm{V} / \mathrm{V}_{0}\right)^{1 / 3}$ should be approximately linear, of slope $=3 / 2\left[(\partial B / \partial P)_{0}-1\right]$ and intercept $=B_{0}$. Where the subscript 0 refers to equilibrium values. We found (Ref. 4) that this is in fact an accurate description of EOS data for many materials from all classes of solids over a broad range of temperatures. This is an experimental confirmation of the expectations expressed above based on the weak volume dependence of $P *(T, V)$.

As further confirmation, we added a volume-independent $P *(T)$ to pressure-volume data (Ref. 6) for cestum of strength sufficient to increase or decrease $v_{0}(T)$ by up to 4 percent. This thermal volume change and corresponding change in thermal pressure is larger than one could actually encounter in solid cesium. Nevertheless, we found that the linearity of plots of. In $H(V, T)$ versus $1-\left(V / V_{0}(T)\right)^{1 / 3}$ was virtually unchanged by the addition of $P \star(T)$. Values of the correlation coefficient indicating the quality of least-mean-square linear fit, varied from 0.99995 for the original 
experimental data to 0.99992 with the largest added $P^{*}(T)$. However, the effect of the added $P *(T)$ was found in changes of corresponding $V_{0}, B_{0}$, and $(\partial B / \partial P)_{0}$ with temperature.

Thus we have a universal form for the EOS of all classes of solids including the effects of temperature. In addition, there is the capability (Ref. 4) to predict EOS, requiring only this single form and input equilibrium data: $V_{0}, B_{0}$, and $(\partial B / \partial P)_{0}$ at each temperature. While this is a relatively small amount of information necessary to predict the EOS, in the next section we will derive a predictive scheme which requires zero pressure data only at a single (reference) temperature.

III. HIGH TEMPERATURE PREDICTIONS FROM REFERENCE TEMPERATURE

\section{ZERO PRESSURE DATA}

The form of the universal EOS allows us to make predictions given $v_{0}$, $B_{0}$, and $(\partial B / \partial P)_{0}$. Here we would like to show that for some solids, high. temperature thermodynamical properties can be predicted via the universal EOS given zero pressure data at a single (reference) temperature. (Note that for metals, the universal binding energy relation, Eq. (2.1), provided a means of predicting the thermal expansion coefficient at a single temperature and zero pressure melting temperatures (Ref. 19)).

The universal form (see discussion following Eq. (2.5) as well as Eq. (4.1)), depends at high temperatures on $P *(T, V)$ having a much weaker dependence on $V$ than - $d E(V) / d V$ has. This characteristic seems to be generally true (see, e.g., Refs. 5 to 8 , and 17). The new assumptions in this paper are that $P *(T, V)$ is independent of $V$ and linearly dependent on $T$ for $T \gtrsim \theta_{D}$ (where $\theta_{D}$ is the Debye temperature).

- Experimentaliy, $P^{\star}(T, V)$ can be very often assumed to be independent of V. Examples are the alkali metals (Ref. 6), solid xenon (Refs. 7 and 17), $\mathrm{NaCl}$, Lif and MgO (see Ref. 8 for the last three). However, for solid argon 
and krypton the volume dependence of $P *(T, V)$ was found (Ref. 7 ) to be not negligible, but still much weaker than that of - $d E(V) / d V$. Thus where appropriate one might assume that

$$
P *(T, V) \rightarrow P *(T)
$$

In addition, it has been observed experimentally that above the Debye temperature, $P^{*}(T, V)$ is a linear function of T (see, e.g., Refs. 6 to 8 and 17). By definition,

$$
\left(\frac{\partial P(T, V)}{\partial T}\right)_{V}=\left(\frac{\partial P^{*}(T, V)}{\partial T}\right)_{V}=\alpha_{0}(T, V) B_{0}(T, V)
$$

where $\alpha_{0}(T, V)$ is the volumic thermal expansion coefficient. Thus (for $T \gtrsim \theta_{0}$ ) linearity with $T$ and Eq. (3.1) imply

$$
P *(T)=P *\left(T_{R}\right)+\alpha_{0}\left(T_{R}\right) B_{0}\left(T_{R}\right)\left(T-T_{R}\right)
$$

where $T_{R} \gtrsim \theta_{D}$ and $T_{R}$ is a reference temperature.

Combining Eqs. (2.4) and (3.3), we have for $T \gtrsim \theta_{0}$,

$$
P(T, V)=P\left(T_{R}, V\right)+\alpha_{0}\left(T_{R}\right) B_{0}\left(T_{R}\right)\left(T-T_{R}\right)
$$

By Eq. (3.4), the high temperature EOS and corresponding thermodynamical properties can be predicted from a knowledge of zero pressure properties at the reference temperature $T_{R}$. Specifically, $P\left(T_{R}, V\right)$ is specified by $B_{0}\left(T_{R}\right), V_{0}\left(T_{R}\right)$ and $(\partial B / \partial P)_{0}\left(T_{R}\right)$ because of the universal form (Ref. 4$)$ of EOS. The only additional input number is $\alpha_{0}\left(T_{R}\right)$.

From Eq. (3.4) we find that the isothermal bulk modulus as given by

$$
B(T, V)=B(V)=-V \frac{d P\left(T_{R}, V\right)}{d V}
$$

and

$$
\left(\frac{\partial B}{\partial P}\right)_{T}(T, V)=-\frac{V}{B(V)} \frac{d B(V)}{d V}
$$

The zero pressure thermal expansion can also be predicted from the EOS, Eq. $(3.4)$, as 


$$
P\left(T_{R}, V\right)+\alpha_{0}\left(T_{R}\right) B_{0}\left(T_{R}\right)\left(T-T_{R}\right)=0
$$

We will now test the predictions from Eqs. (3.4 to 3.7), making use (Ref. 4) of the universal EOS for the reference isotherm, $P\left(T_{R}, V\right)$.

IV. ANALYTIC APPROXIMATION TO UNIVERSAL EQUATION OF STATE

The universal EOS has been described in Ref. 4 and Section II. It was pointed out there that plots of $\ln H(V, T)$ (Eq. (2.5)) versus (1 $\left.\left(V / V_{0}(T)\right)^{1 / 3}\right)$ were approximately linear at each temperature, with slope and intercept yielding $B_{0}(T)$ and $(\partial B / \partial P)_{0}(T)$. Thus a simple, analytic approximation (Ref. 4) to the universal EOS is:

$$
P(T, X)=\frac{3 B_{0}(T)}{x^{2}}(1-x) \exp \left[n_{0}(T)(1-x)\right]
$$

where

$$
x \equiv\left(\frac{V}{v_{0}(T)}\right)^{1 / 3}
$$

and

$$
\eta_{0}(T)=\frac{3}{2}\left[\left(\frac{\partial B}{\partial P}\right)_{0}(T)-1\right]
$$

This analytic form, Eq. (4.1), is particularly convenient when used as the reference isotherm since it allows one to make analytic predictions of high temperature thermodynamical properties through Eqs. (3.4) to (3.7). In terms of Eq. (4.1), Eqs. (3.4) to (3.7) become, respectively,

$$
\begin{aligned}
& P(T, X)=\frac{3 B_{0}\left(T_{R}\right)}{x^{2}}(1-x) \exp \left[n_{0}\left(T_{R}\right)(1-x)\right]+\alpha_{0}\left(T_{R}\right) B_{0}\left(T_{R}\right)\left(T-T_{R}\right) \\
& B(T, X)=\frac{B_{0}\left(T_{R}\right)}{x^{2}}\left[2+\left(\eta_{0}\left(T_{R}\right)-1\right) x-\eta_{0}\left(T_{R}\right) x^{2}\right] \exp \left[\eta_{0}\left(T_{R}\right)(1-x)\right]
\end{aligned}
$$


and

$$
\left(\frac{\partial B}{\partial P}\right)(T, X)=\frac{4+\left(3 n_{0}\left(T_{R}\right)-1\right) x+n_{0}\left(T_{R}\right)\left(n_{0}\left(T_{R}\right)-1\right) x^{2}-n_{0}^{2}\left(T_{R}\right) x^{3}}{3\left(2+\left(n_{0}\left(T_{R}\right)-1\right) x-n_{0}\left(T_{R}\right) x^{2}\right)}
$$

where; for Eqs: (4.4) to:(4.6),

$$
x=\left(\frac{V}{V_{0}\left(T_{R}\right)}\right)^{1 / 3}
$$

It is appropriate now to compare Eqs. (4.1) and (4.4). As discussed in Section III, Eq. (4.4) is an approximation to Eq. (4.1) which is reasonable for $T \gtrsim \theta_{D}$ and for those solids for which Eq. (3.1) is a good approximation. The numerical test described in the next to last paragraph of Section II suggeşts that Eqs. (4.1) and (4.4) are consistent with each other to a good approximation. As discussed earlier; the advantage of Eq. (4.4) is that values of $B_{0}(T), \alpha_{0}(T)$, and $(\partial B / \partial P)_{0}(T)$ are required only at $T=$ $T_{R}$, while for Eq. (4.1) $V_{0}(T), B_{0}(T)$ and $(\partial B / \partial P)_{0}(T)$ are required at a) $T$ of interest.

\section{COMPARISON WITH EXPERIMENT}

We will now compare predictions of Eqs. (3.4) to (3.7) with experimental data. The analytic approximation to the unfversal. EOS will be used for the reference isotherm, Eqs. (4.4) to (4.6). Comparison will also be made, where appropriate, with use of the Murnaghan (Ref. 20) EOS for the reference isotherm in Eqs. (3.4) to (3.7). The Murnaghan EOS, which is extensively used (see, e.g., Refs, 21 and 22), has decreasing accuracy:(Refs. 4 and 23) as compression increases.

As the universal EOS applies to all classes of solids, we carried out this test for representatives from three classes of solids. These are gold, sodium chloride, and xenon, exhibiting respectively metallic, ionic, and rare-gas bonding. 


\section{A. Input Parameters}

Equations (4.4) to (4.6) require only three input parameters for each solid. These are listed in Table I for the three representative solids, along with selected reference and Debye temperatures.

A few comments must be made: for sodium chloride and xenon, the reference temperatures have been chosen at temperatures slightly lower than the Debye temperatures. This was necessary due to the very limited EOS experimental data, and is not expected to introduce important errors in the predictions of the model. For gold and sodium chloride the values of $B_{0}\left(T_{R}\right)$ and $(\partial B / \partial P)_{0}\left(T_{R}\right)$ have been chosen as the average of values. taken from the references indicated in Table $I$.

It is difficult to estimate $(\partial B / \partial P)_{0}\left(T_{R}\right)$ for gold from the 1iterature. Consequently, we will examine the predictions of our theory for two different values: the lowest one represents most of the static pressure experiments $\left((\partial B / \partial P)_{0}\left(T_{R}\right)=5.5\right)$ and the highest value represents most of the ultrasonic measurements $\left((\partial B / \partial P)_{0}\left(T_{R}\right)=6.5\right)$ (Ref. 24). Values for xenon have been taken from the analysis carried out by Birch (Ref. 25) on data obtained by M.S. Anderson and C.A. Swenson (Ref. 7). Finally, thermal expansion data have been taken from the complete compilations in Refs. 26 and 27 for gold and sodium chloride respectively. The value for xenon has been taken from the study of Manzhelil et al. (Ref. 28).

\section{B. Prediction of High Temperature Isotherms}

In this section we will estimate high temperature isotherms near the melting point from input data measured for $T \approx \theta_{0}$. The results are then compared with high temperature isotherms taken from the literature. Figure 1 compares the results for gold with the estimates of Heinz and Jeanloz (Ref. 24). Their estimates are not direct experimental data but extrapolations of the considered isotherms using a Mie-Gruneisen equation. 
Heinz and Jeanloz estimate that their results contain a 1 to 2 percent error in the pressure. A problem arises from uncertainties in the value of $(\partial B / \partial P)_{0}\left(T_{R}\right)$. In their analysis of experimental data, Heinz and Jeanloz find a value close to 5.5: however, most ultrasonic measurements give values around 6.5 (Refs. 24 , and 29 to 31 ). It is interesting to note that a fitting of the room temperature EOS proposed in Ref. 24 to our universal EOS (Eq. (4.4)) would give a value of 5.8 . In the present analysis, we show the results for the two extreme values of 5.5 and 6.5 for $(\partial B / \partial P)_{0}\left(T_{R}\right)$ for gold (Fig. 1). The agreement is good over the entire range of compression and is considerably better than that obtained using the Murnaghan (Refs. 20 and 22) EOS for the reference isotherm in Eqs. (3.4) to (3.7).

In Fig. 2 we compare the results for $\mathrm{NaCl}$ with the experimental data of Boehler and Kennedy (Ref. 32) and with data representative of the Decker model (Ref. 33). Again, the prediction via Eq. (4.4) is relatively accurate. The prediction based on the Murnaghan reference isotherm is again accurate at low pressures but becomes increasingly inaccurate as the pressure increases as is to be expected.

Finally, solid xenon, as shown in Fig. 3 , confirms the results of both Figs. 1 and 2. Again the predictions based on Eq. (4.4) give good agreement When compared to experimental data (M.S. Anderson and C.A. Swenson (Ref. 7)), while that based on the Murnaghan $P\left(T_{R}, V\right)$ deviate significantly at high compression.

For the three different materials investigated so far, we can conclude that the use of the universal description of the reference isothermal EOS combined with the assumptions of Section III gives an accurate prediction of the high temperature EOS. We have also demonstrated that the Murnaghan EOS cannot describe the reference isotherm well enough to allow extrapolations to high temperatures when substantial compression occurs. 


\section{Prediction of Thermal Expansion}

In Section III, a method was described for obtaining the thermal expansion curve given the reference temperature isotherm (Eq. (3.7)). In this section, thermal expansion predictions will be compared with experiment. For simplicity, we will limit our study to zero pressure thermal expansion (Eq. (3.7)) for which experimental data are easily avaliable in the 1iterature. However, we note that the same approach predicts a at high pressure $(P(V, T) \neq 0$ in Eq. (3.4)), where data are generally not available. We will use the universal EOS for the reference isotherm, so that from Eq. (4.4)

$$
\frac{3}{x^{2}}(1-x) \exp \left[n_{0}\left(T_{R}\right)(1-x)\right]+\alpha_{0}\left(T_{R}\right)\left(T-T_{R}\right)=0
$$

Here $X$ is defined by Eq. (4.7) and $n_{0}\left(T_{R}\right)$ by Eq. (4.3) evaluated at $T_{R}$. Thus the zero pressure thermal expansion is determined entirely by $\alpha_{0}\left(T_{R}\right), V_{0}\left(T_{R}\right)$, and $(\partial B / \partial P)_{0}\left(T_{R}\right)$.

For cubic solids, this function of volume can be reduced to a function of length. Figures 4 to 6 are plots of the predicted values of thermal expansion along with experimental data for gold, sodium chloride, and solid xenon. The two input parameters can be found in Table I. Also for comparison the linear expansion $\alpha_{0}\left(T_{R}\right)\left(T-T_{R}\right) / 3$ is shown.

A common feature of Figs. 4 to 6 is that Eq. (5.1) gives a good prediction of thermal expansion for temperatures ranging from the Debye temperature up to the melting point. The nonlinear behavior of the thermal expansion is well reproduced. This is due primarily to the universal EOS. Such nonlinear dependence on temperature has been difficult to predict (Ref. 9).

A second common result is that Eq. (5.1) does not accurately predict the thermal expansion for temperatures less than the Debye temperature. Recall 
that Eq. (3.3) is valid only for $T \geqslant \theta_{0}$ and that Eq. (3.3) was assumed in the derivation of $(5.1)$. Equations (3.1) and (3.3) require that $\alpha_{0}(T, V)$ $B_{0}(T, V)$ be independent of $T$ and $V$. Since the thermal expansion coefficients goes to zero at zero temperature, $\alpha_{0}(T, V) B_{0}(T, V)$ also $\rightarrow 0$ as $T . \rightarrow 0$. Thus it is to be expected that predictions from Eq. (5.1) would not be accurate for low temperatures.

In Fig. 4 we investigated the sensitivity to the input parameter, $(\partial B / \partial P)_{0}\left(T_{R}\right)$. Using the two extreme values for gold of 5.5 and 6.5 , the maximum deviation between predicted thermal expansion of gold is only 3 percent of . $\Delta L / L\left(T_{R}\right)$.

However, Eq. (5.1) is slightly more sensitive to the choice of the other parameter, $\alpha_{0}\left(T_{R}\right)$. As shown in Fig. 5 , a change of about 2.5 percent in $\alpha_{0}\left(T_{R}\right)$, consistent with experimental accuracy $\left(\alpha_{0}\left(T_{R}\right)=1.2 \times 10^{-4}\right.$ $K^{-1}$ in Ref. 27 and $1.17 \times 10^{-4} K^{-1}$ in Ref. 32 for the same temperature of. $300 \mathrm{~K}$ ), brings maximum variations of the predicted values of about 4 percent of $\Delta L / L\left(T_{R}\right)$.

\section{Temperature Dependence of the Bulk Modulus}

In Section III we assumed that the isothermal bulk modulus has no explicit dependence on the temperature and is a function of the volume only (Eq. (3.5)). In Section IV, we proposed an analytical form of this function $B(V)$, based on the universal EOS (Eq. (4.5)). We can now use the predictions of the thermal expansions obtained previousiy (Eq. (5.1)) to compute the temperature induced volume changes and consequently, the corresponding changes of isothermal buik moduius. This computation has been done for gold, sodium chloride, and solid xenon and the results are plotted in Figs. 7 to 9 , respectively. Again, the only input parameters are the three listed in Table I. 
The results achieved with our model agree well with available experimental data over the investigated temperature range. In addition to these general comments, Fig. 7 shows that, for gold, the choice of an input value of 6.5 for $(\partial B / \partial P)_{0}\left(T_{R}\right)$, which corresponds to ultrasonic measurements, gives the best results. This was to be expected since the experimental data used here to test the bulk modulus prediction (from Refs. 30 and 36) are also obtained from ultrasonic measurements.

In the case of xenon, where low temperature experimental data are available (Ref. 25), Fig. 9 shows a deviation of the predicted values from the experimental values for temperatures close to $0 \mathrm{~K}$. This was also to be expected since the approximations made in Section III do not adequately represent the low temperature behavior of solids. The slope of the curves $B_{0}(T)$ must be zero at zero temperature on general thermodynamic grounds. This is confirmed by the experimental data for xenon, but is not represented by our model.

\section{E. Temperature Dependence of $(\partial B / \partial P)_{0}$}

The same approach used for the isothermal bulk modulus can be used for $(\partial B / \partial P)_{0}$ since this quantity is also assumed to have no explicit temperature dependence (Eq. (3.6)). We use Eq. (4.6) and the previously calculated values of thermal expansion (Eq. (5.1)) for sodium chloride and xenon to obtain a prediction of the thermal behavior of $(\partial B / \partial P)_{0}$.

Figures 10 and 11 present respectively the results for sodium chloride and xenon, obtained by using the three input parameters listed in Table I. These results are compared with experimental data from ultrasonic measurements (sodium chloride) or from analysis of high pressure EOS data (xenon). Unfortunately, to our knowledge, no similar high temperature set of data exists for gold. 
For sodium chloride, the agreement between the prediction of Eq. $(4.6)$ and the experimental data, as presented in $\mathrm{Fig} .10$, is good. For xenon (Fig. 11), agreement is worse. However, the accuracy of the experimental data which is extrapolated from static high pressure measurements is questionable. For example, the two sets of data presented in Fig. 11 are extrapolated from the same set of high pressure experiments, using two different methods (Refs. 7 and 25): As a consequence, it is clear that one must add the uncertainties of the high pressure measurements to the uncertainty caused by the extrapolation method used to obtain the values of $(\partial B / \partial P)_{0}$. Therefore, a check of our predictions of the thermal variations of $(\partial B / \partial P)_{0}$ will require more complete and accurate sets of experimental data.

VI. SUMMARY

We have discussed a recently proposed "universal" EOS which accurately describes isothermal P-V data for all classes of solids in compression. Phase transitions were excluded from the analysis. Results from Ref. 4 show that the EOS can be predicted from the universal relation if $V_{0}(T), B_{0}(T)$ and $(\partial B / \partial P)_{0}(T)$ are known for the temperatures of interest.

In this paper we have used the fact that an additional assumption is often valid. Namely it was assumed that the thermal pressure is independent of $V$ and varies linearly with $T$ for $T \geqslant \theta_{0}$. With solids for which this is an accurate approximation we showed that high temperature thermodynamical properties can be predicted from $\alpha_{0}\left(T_{R}\right), V_{0}\left(T_{R}\right)$, ${ }_{0}{ }_{0}\left(T_{R}\right)$ and $(\partial B / \partial P)_{0}\left(T_{R}\right)$, where $T_{R}$ is a single reference temperature. An analytic approximation was introduced for the universal EOS. This led to a number of simple formulas characterizing the high temperature EOS in the absence of phase transitions. Simple expressions of particular interest were given for: (1) the high temperature 1sotherms; (2) the temperature dependence of the thermal expansion for a given $P$; and (3) the 
temperature dependence of the $P=0$ bulk modulus and its pressure derivative. These simple expressions were compared with experiment for gold, sodium chloride, and xenon. Good agreement between prediction and experiment was obtained for the high temperature isotherms, as well as the temperature dependence of the thermal expansion, bulk modulus, and pressure derivative of the bulk modulus for gold, sodium chloride, and xenon.

Finally, we want to emphasize the wide generality of a model which describes the high temperature thermodynamic behavior of a metal and an alkali halide as well as a rare gas solid, from $P=0$ input data at a single (reference) temperature.

\section{ACKNOWLEDGMENT}

One of us (P.V.) would like to thank the French "Ministère des Relations Exterieures" for providing him a grant. The Ames Laboratory is operated for the U.S. Department of Energy by Iowa State University under contract number W-7405-ENG-82 and this work was supported by the Director of Energy Research, U.S. Office Basic Energy Sciences.

\section{REFERENCES}

1. Godwal, B.K.; Sikka, S.K.; and Chidambaram, R.: Equation of State Theories of Condensed Matter up to about $10 \mathrm{TPa}$. Phys. Rep., vol. 102, no. 3, Dec. 1983, pp. 121-197.

2. Zharkov, V.N.; and Kalinin, V.A.: Equations of State for Solids at High Pressures and Temperatures. Consultants Bureau-Plenum, 1971.

3. Ruoff, A.L.; and Chhabildas, L.C.: Status of Equation of State of Solids. High Pressure Sclence and Technology, Vol. 1, K.D. Timmerhaus and M.S. Barber, Plenum, 1979, pp. 19-32.

4. Vinet, P., et al.: Universality in the Compressive Behavior of Solids. NASA TM-87303, 1986 unpublished. 
5. Swenson, C.A.: Equation of State of Cubic Solids; Some Generalizations. J. Phys. Chem. Solids, vol. 29, no. 8, Aug. 1968, pp. 1337-1348.

6. Anderson, M.S.; and Swenson, C.A.: Experimental Equations of State for Cesium and Lithium Metals to $20 \mathrm{kbar}$ and the High-Pressure Behavior of the Alkali Metals. Phys. Rev. B, vol. 31, no. 2, Jan. 15, 1985, pp. 668-680.

7. Anderson, M.S.; and Swenson, C.A.: Experimental Equations of State for the Rare Gas Solids. J. Phys. Chem. Solids, vol. 36, no. 3, Mar. 1975, pp. $145-162$.

8. Anderson, 0.L.; Boehler, R.; and Sumino, Y.: Anharmonicity in the Equation of State at High-Temperature for some Geophysically Important Solids. High Pressure Research in Geophysics, S. Akimoto and M.H. Manghnani, eds., Center for Academic Publications, Tokyo, Japan, 1982, pp. $273-284$.

9. MacDonald, R.A.; and Shulka, R.C.: Thermodynamic Properties of bcc Crystals at High Temperatures: The Transition Metals. Phys. Rev. B, vol. 32 , no. 8 , Oct. 15,1985, pp. 4961-4968.

10. Ferrante, J.; and Smith, J.R.: Theory of Bimetallic Interface. Phys. Rev. B, vol. 31, no. 6, Mar. 15, 1985, pp. 3427-3434.

11. Smith, J.R.; Ferrante, J.; and Rose, J.H.: Universal Binding-Energy Relation in Chemisorption. Phys. Rev. B, vol. 25, no. 2, Jan. 15, 1982, pp. 1419-1422.

12. Rose, J.H., Smith, J.R., and Ferrante, J.: Universal Features of Bonding in Metals. Phys. Rev. B, vol. 28, no. 4, Aug. 15, 1983, pp. 1835-1845.

13. Rose, J.H.; Vary, J.P.; and Smith, J.R.: Nuclear Equation of State from Scaling Relations for Solids. Phys. Rev. Let., vol. 53, no. 4, July 23, 1984, pp. 344-347. 
14. Smith, J.R., et a1.: Universal Features of Bonding-Energy as a Function of Interatomic Spacing. Many-Body Phenomena at Surfaces, D. Langreth and H. Suh1, eds., Academic Press, 1984, pp. 159-174.

15. Harrison, W.A.: Electronic Structure and the Properties of Solids. W.H. Freeman and Co., 1980. (especially Appendix C).

16. C.A. Swenson and M.S. Anderson: Piston - Displacement Equations of State for $\mathrm{Na}, \mathrm{K}$, Rb Metals to 20 kilobar from $300 \mathrm{~K}$ to $4 \mathrm{~K}$, in High Pressure in Science and Technology Part III - Materials Research Soc. Symposium Proceedings Vol. 22, Eds. C. Homan, R.K. McCrone, and E. Whalley, North Holland Publishing Co. 1984, New York, Amsterdam, Oxford - pp. 251-254.

17. Packard, J.R.; and Swenson, C.A.: An Experimental Equation of State for Solid Xenon. J. Phys. Chem. Solids, vol. 24, 1963, pp. 1405-1418.

18. Rose, J.H., et al.: Universal Features of the Equation of State of Metals. Phys. Rev. B, vol. 29, no. 6, Mar. 15, 1984, pp. 2963-2969.

19. Guinea, F., et al.: Scaling Relations in the Equation of State, Thermal Expansion, and Melting of Metals. Appl. Phys. Lett., vol. 44, no. 1, Jan. 1,1984, pp. 53-55.

20. Murnaghan, F.D.: Finite Deformation of an Elastic Solid. Chapter 4, Wiley, 1951.

21. Dass, N.; and Kamuri, M.: Derivation of some Equations of State for Solids. Phys. Stat. Sol. B, vol. 127, 1985, Pp. 103-108.

22. Yin, M.T.; and Cohen, M.L.: Theory of Static Structural Properties, Crystal Stability, and Phase Transformations: Application to St and Ge. Phys. Rev. B, vol. 26, no. 10, Nov. 15, 1982, pp. 5668-5687.

23. Anderson, 0.L.: The use of Ultrasonic Measurements Under Modest Pressure to Estimate Compression at High Pressure. J. Phys. Chem. Solids, vol. 27 , no. 3, Mar. 1966, pp. 547-565. 
24. Heinz, O.L.; and Jean Ioz, R.: The Equation of State of the Gold Calibration Standard. J. Appl. Phys., vol. 55, no. 4, Feb. 15, 1984, pp. 885-893.

25. Birch, F.: Isotherms of the Rare Gas Solids. J. Phys. Chem. Solids, vol. 38, no. 2, 1977, pp. 175-177.

26. Touloukian, Y.S., et al.: Thermophysical Properties of Matter, Vol. 12 Thermal Expansion, Metalilic Elements and Alloys, IFI/Plenum, 1975.

27. Touloukian, Y.S., et al.: Thermophysical Properties of Matter, Vol. 13 Thermal Expansion, Non Metallic Solids, IFI/Plenum, 1977.

28. Manzheli1, V.G.; Gavrliko,V.G.; and Voitovich, E.I.: Thermal Expansion of Solidified Rare Gases. Phys. Stat. Sol., vol. 17, 1966, Pp. K139-K141.

29. Daniels, W.B.; and Smith, C.S.: Pressure Derivitives of the Elastic Constants of Copper, Sllver, and Gold to 10000 Bars. Phys. Rev., vol. 111, no. 3, Aug. 1, 1958, pp. 713-721.

30. Biswas, S.N.; Van't Klooster, P.; and Trappeniers, N.J.: Effect of Pressure on the Elastic Constants of Noble Metals from -196 to $+25{ }^{\circ} \mathrm{C}$ and up to 2500 bar. Physica B, vol. 103B, 1981, pp. 235-246.

31. Barsch, G.R.; and Chang, Z.P.: Adiabatic, Isothermal, and Intermediate Pressure Derivitives of the Elastic Constants for Cubic Symmetry. Phys. Stat. Sol., vol. 19, 1967, pp. 139-151.

32. Boehler, R.; and Kennedy, G.C.: Equation of State of Sodium Chloride up to $32 \mathrm{kbar}$ and $500{ }^{\circ} \mathrm{C}$. J. Phys. Chem. Solids, vol. 41, no. 5, 1980, pp. 517-523.

33. Decker, D.L.: High-Pressure Equation of State for $\mathrm{NaCl}, \mathrm{KCl}$, and $\mathrm{CsCl}$. J. App1. Phys., vol. 42, no. 8, July 1971, pp. 3239-3244.

34. Tilford, C.R.; and Swenson, C.A.: Thermal Expanstons of Solid Argon, Krypton, and Xenon Above 1K. Phys. Rev. B, vol. 5, no. 2, Jan. 15, 1972, pp. $719-732$. 
35. Gavrilko, V.G.; and Manzhe11i, V.G.: Density of Crystalline Xenon. Sov. Phys. - Solid State, vol. 6, no. 7, Jan. 1965, pp. 1734-1735.

36. Chang, Y.A.; and Himme1, L.: Temperature Dependence of the Elastic Constants of $\mathrm{Cu}, \mathrm{Ag}$, and Au Above Room Temperature. J. Appl. Phys., vol. 37, no. 9, Aug. 1966, pp. 3567-3572.

37. Speltzer, H.; Sammis, C.G.; and 0'Connell, R.J.: Equation of State of $\mathrm{NaCl:}$ Ultrasonic Measurements to $8 \mathrm{kbar}$ and $800{ }^{\circ} \mathrm{C}$ and Static Lattice Theory. J. Phys. Chem. Solids, vol. 33, no. 9, Sept. 1972, pp. 1727-1750.

38. Barte15, R.A.; and Schuele, D.E.: Pressure Derivitives of the Elastic Constants of $\mathrm{NaCl}$ and $\mathrm{KCl}$ at $295 \mathrm{~K}$ and $195 \mathrm{~K}$. J. Phys. Chem. Solids, vol. $26,1965, \mathrm{pp} .537-549$. 
TABLE I. - SUMMARY OF THE THREE INPUT PARAM-

ETERS AS WELL AS DEBYE TEMPERATURES

$\theta_{D}$ AND REFERENCE

TEMPERATURES $T_{R}$

\begin{tabular}{|c|c|c|c|}
\hline Input data & Gold & $\mathrm{NaCl}$ & Xenon \\
\hline $\begin{array}{l}B_{0}\left(T_{R}\right)(1010 \mathrm{~Pa}) \\
\alpha_{0}\left(T_{R}\right)(10-5 K-1) \\
(\partial B / \partial P)_{0}\left(T_{R}\right)\end{array}$ & $\begin{array}{l}\text { a } 16.6 \\
\text { d } 4.25 \\
\text { a } 5.5-6.5\end{array}$ & $\begin{array}{l}\text { b2.35 } \\
\text { e12.0 } \\
\text { b5 } 5.35\end{array}$ & $\begin{array}{l}c 0.302 \\
f 60.0 \\
c 7.8\end{array}$ \\
\hline \multicolumn{4}{|l|}{ Temperatures } \\
\hline $\begin{array}{l}T_{R}(K) \\
\theta_{D}(K) g\end{array}$ & $\begin{array}{l}300 \\
162\end{array}$ & $\begin{array}{l}298 \\
320\end{array}$ & $\begin{array}{l}60 \\
64\end{array}$ \\
\hline
\end{tabular}

a Reference 24.

b Reference 4 .

c Reference 25.

d Reference 26, data taken at $293 \mathrm{~K}$.

e Reference 27 , data taken at $293 \mathrm{~K}$.

$f$ Reference 28 .

$g$ Reference 3. 


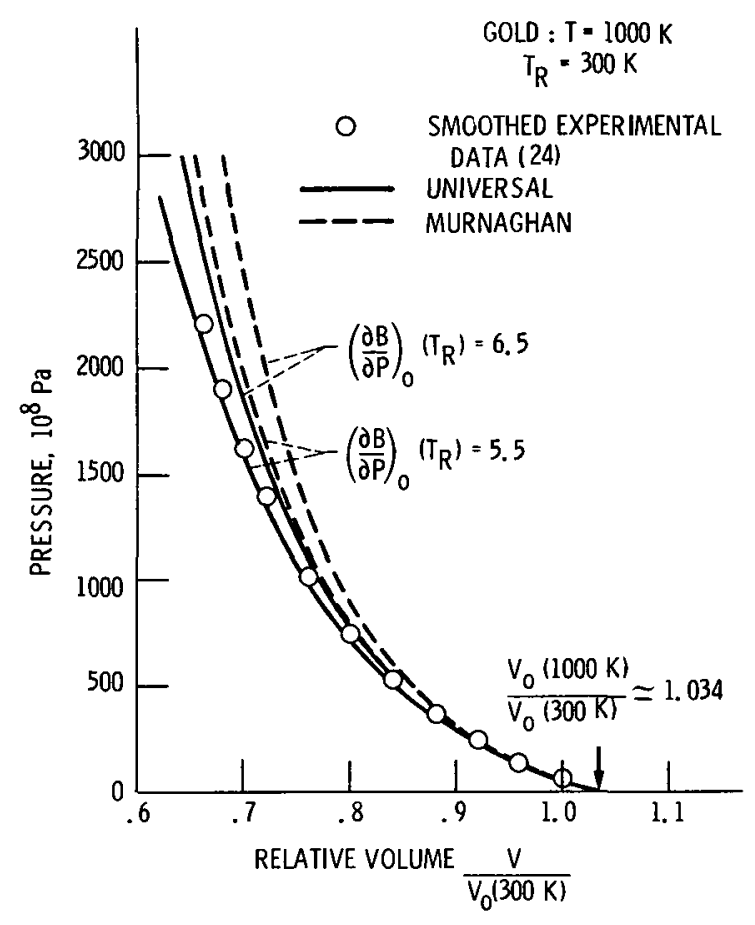

Figure 1. - Prediction of a high temperature $P(T, V)$ isotherm: Gold at $1000 \mathrm{~K}$. Comparison of prediclions based on universal $P\left(T_{R}, V\right)$ (eq. (4.4)) and on the Murnaghan model for $P\left(T_{R}, V\right)$ with experimental data from Heinz and Jeanloz (ref. 24).

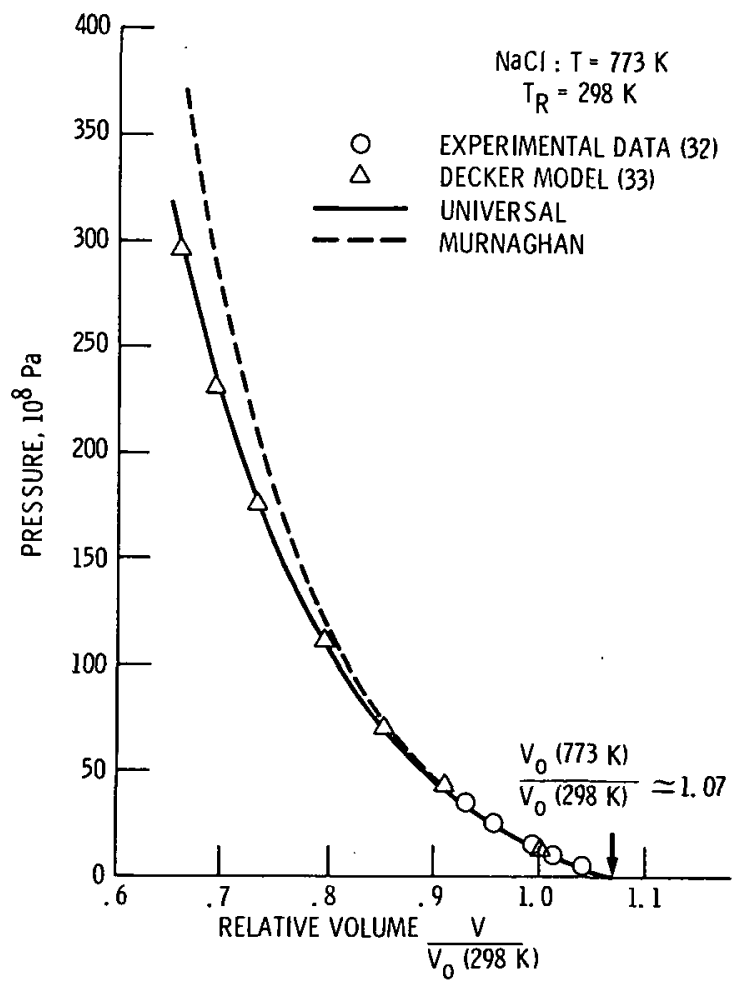

Figure 2. - Prediction of a high temperature $P(T, V)$ isotherm: $\mathrm{NaCl}$ at $773 \mathrm{~K}$. Comparison of predictions based on universal $P\left(T_{R}, V\right)$ (eq. (4. 4)) and on the Murnaghan model for $P\left(T_{R}, V\right)$ with experimental data from Boehler and Kennedy (ref, 32) and representative points from the Decker EOS (ref. 33). 


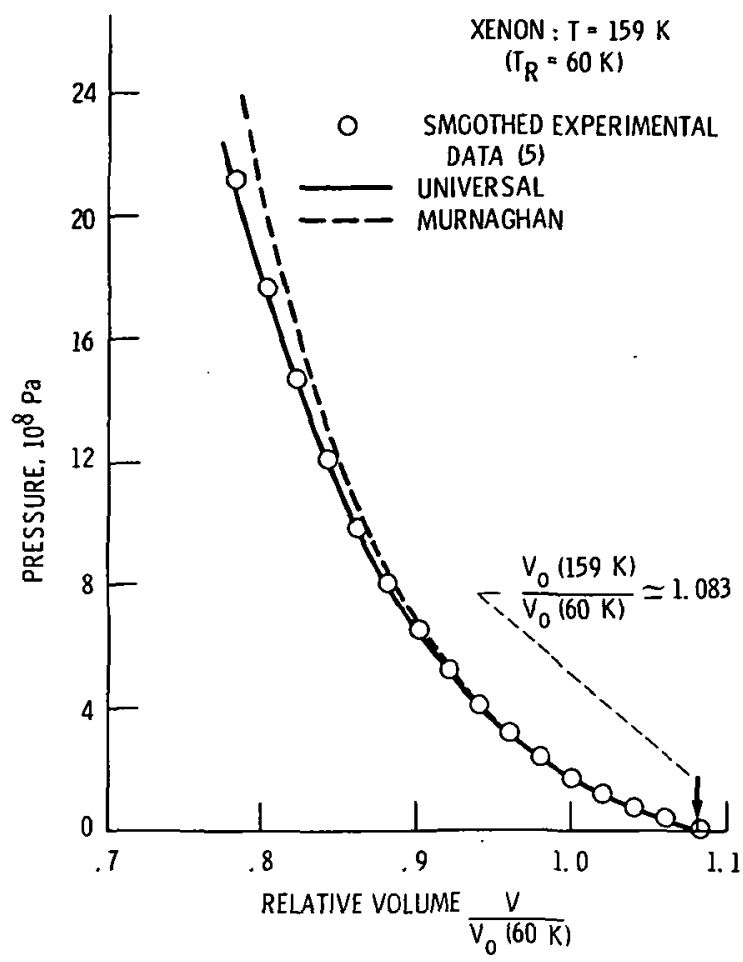

Figure 3. - Prediction of a high temperature $P(V)$ isotherm: Xenon at $159 \mathrm{~K}$. Comparison of predictions based on universal $P\left(T_{R}, V\right)$ (eq. $(4,4)$ ) and on the Murnaghan model for $P\left(T_{R}, V\right)$ with experimental data from M.S. Anderson and Swenson (ref. 7).

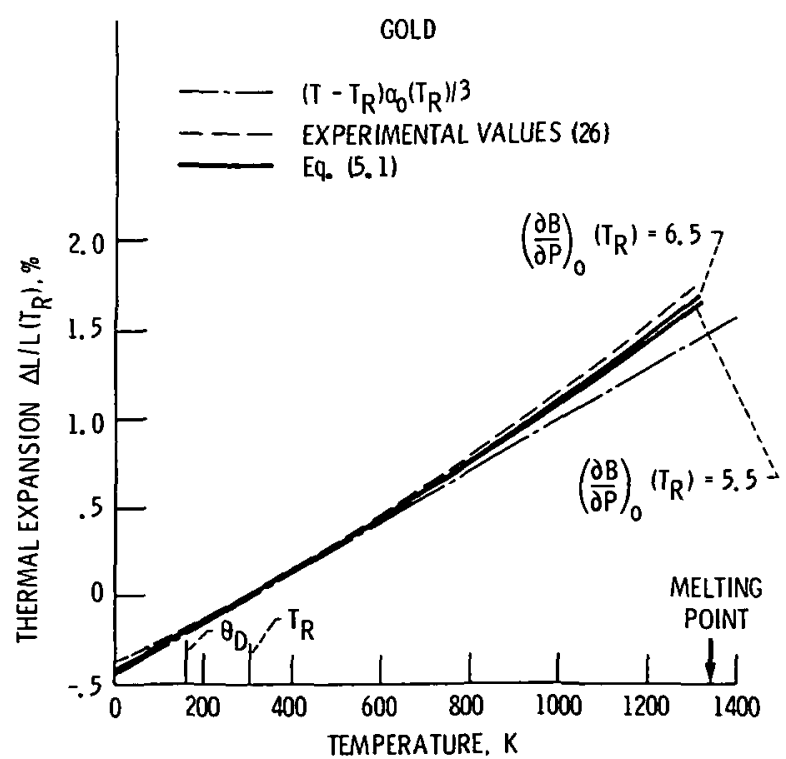

Figure 4. - Prediction of thermat expansion of gold: Plot of prediction from equation (5. 1) with smoothed experimental data from reference 26 . To examine how well nonlinear expansion is predicted, the linear result is also plotted as $\left(T-T_{R}\right) q_{0}\left(T_{R}\right) / 3$. 


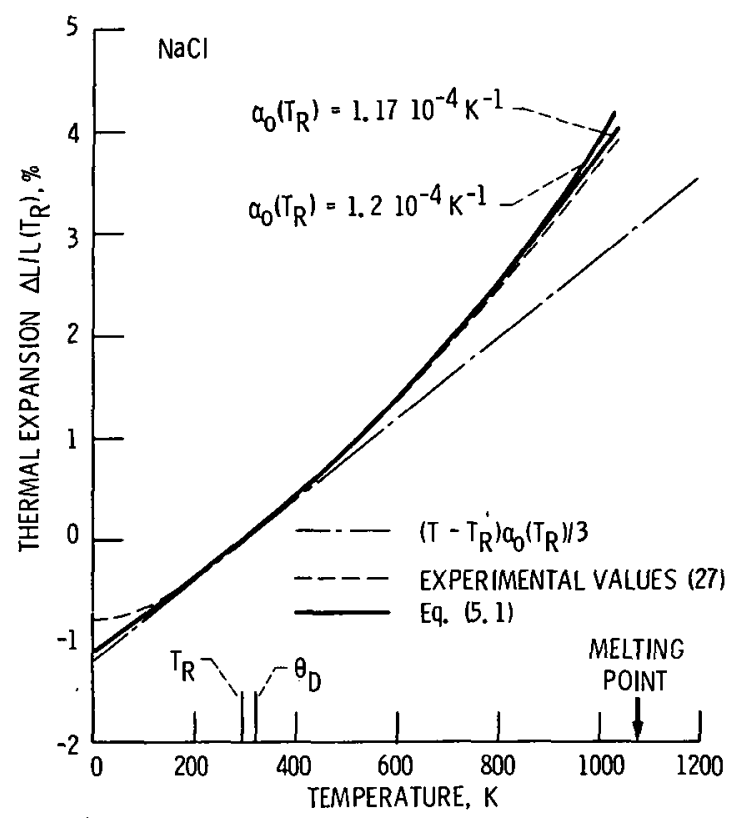

Figure 5. - Prediction of thermal expansion of sodium chloride plotted as in figure 4 with smoothed experimental data from reference 27 .

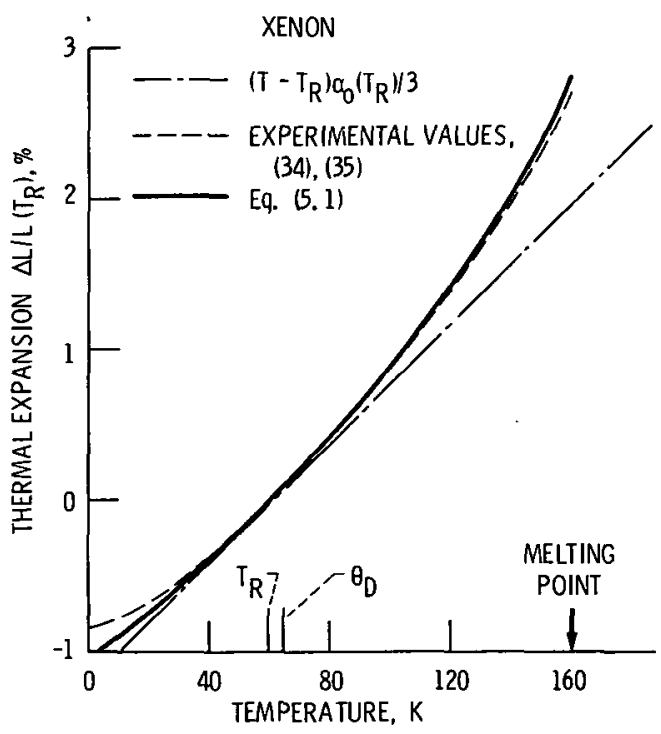

Figure 6. - Prediction of thermal expansion of solid xenon plotted as in figure 4 with experimental data from reference $34(T \leq 100 \mathrm{~K})$ and refarence 35 (T) $100 \mathrm{~K}$ ). (See also ref. 7.) 


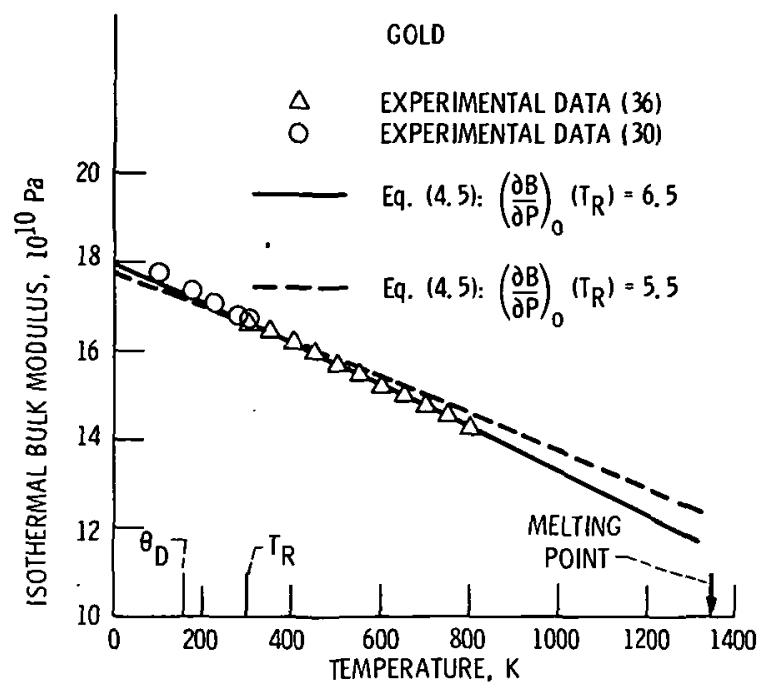

Figure 7. - Prediction of temperature dependence of the isothermal bulk modulus of gold. Plot of the present model (eqs. (4.5) and (5.1)) with experimental data from references 30 and 36 .

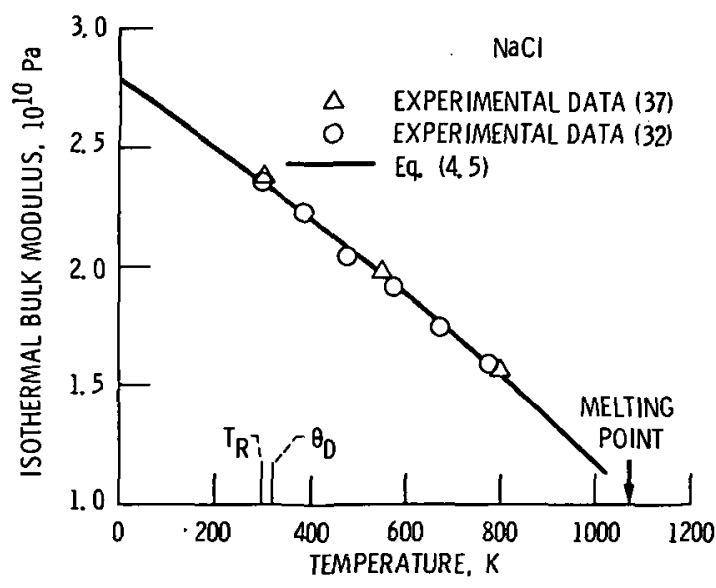

Figure 8. - Prediction of temperature dependence of the isothermal bulk modulus of sodium chlorice: Plot of the present model (eqs. (4.5) and (5.1) with experimental data from references 32 and 37. 


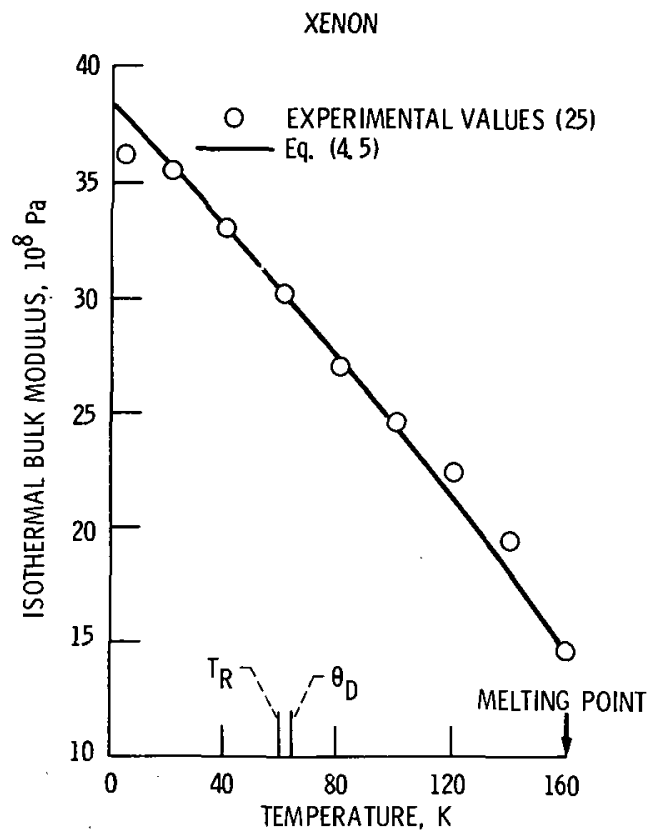

Figure 9. - Prediction of temperature dependence of the isothermal bulk modulus of solid xenon: Plot of the present model (eqs. (4.5) and (5.1)) with experimental data from reference 25 .

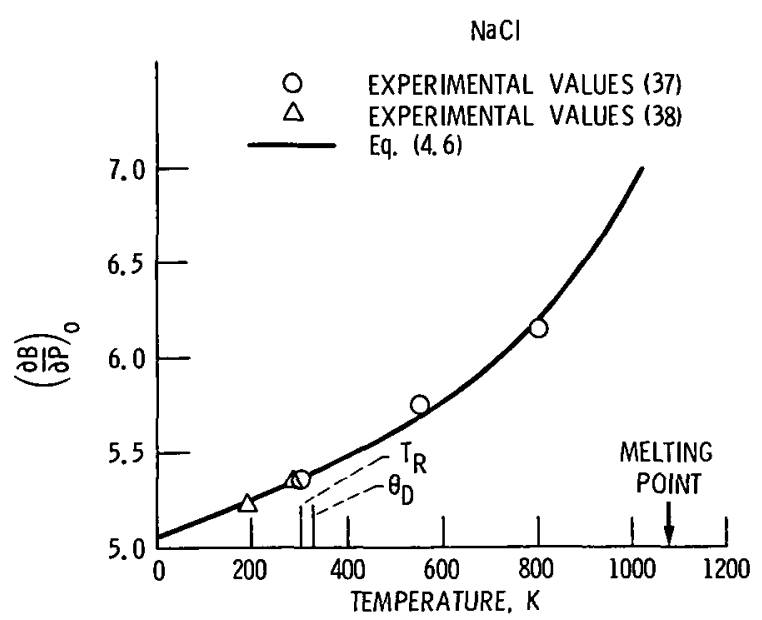

Figure 10. - Prediction of temperature dependence of $\left(\partial B_{0}(T, P) / \partial P\right)_{T}$ for sodium chloride: Plot of the present model (eqs. (4.6) and (5.1) with experimental data from references 37 and 38 . 


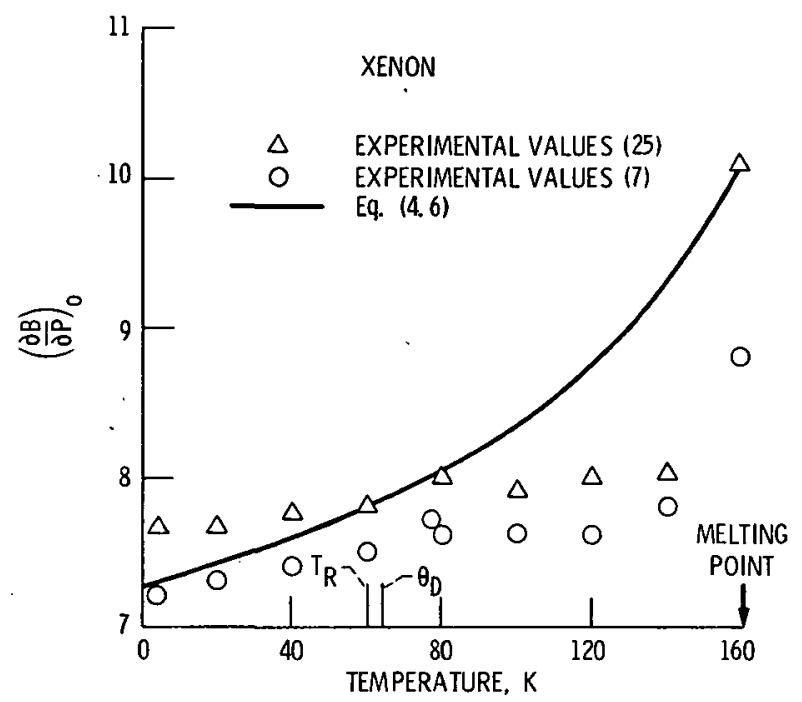

Figure 11. - Prediction of temperature dependence of $\left(\partial \mathrm{B}_{0}(T, P) / \partial P\right)_{T}$ for solid xenon: Plot of the present model (eas. (4.5) and (5.1)) with experimental data extrapolated from high pressure measurements in references 7 and 25 . 


\begin{tabular}{|c|c|c|}
\hline $\begin{array}{l}\text { 1. Report No. } \\
\text { NASA TM-87321 }\end{array}$ & 2. Government Accession No. & 3. Recipient's Catalog No. \\
\hline \multirow{2}{*}{\multicolumn{2}{|c|}{$\begin{array}{l}\text { 4. Title and Subtitle } \\
\text { Temperature Effects on the Universal Equation of } \\
\text { State of Solids }\end{array}$}} & $\begin{array}{l}\text { 5. Report Date } \\
\text { April } 1986\end{array}$ \\
\hline & & $\begin{array}{l}\text { 6. Performing Organization Code } \\
506-53-12\end{array}$ \\
\hline \multirow{2}{*}{\multicolumn{2}{|c|}{$\begin{array}{l}\text { 7. Author(s) } \\
\text { Pascal Vinet, John Ferrante, John R. Smith, and } \\
\text { James H. Rose }\end{array}$}} & $\begin{array}{l}\text { 8. Performing Organization Report No. } \\
\qquad-3001\end{array}$ \\
\hline & & 10. Work Unit No. \\
\hline \multirow{3}{*}{\multicolumn{2}{|c|}{$\begin{array}{l}\text { 9. Performing Organization Name and Address } \\
\text { National Aeronautics and Space. Administration } \\
\text { Lewis Research Center } \\
\text { Cleveland, Ohio } 44135\end{array}$}} & \\
\hline & & 11. Contract or Grant No. \\
\hline & & 13. Type of Report and Period Covered \\
\hline \multicolumn{2}{|l|}{ 12. Sponsoring Agency Name and Address } & Technical Memorandum \\
\hline \multicolumn{2}{|c|}{$\begin{array}{l}\text { National Aeronautics and Space Administration } \\
\text { Washington, D.C. } 20546\end{array}$} & 14. Sponsoring Agency Code \\
\hline \multicolumn{3}{|c|}{$\begin{array}{l}\text { 15. Supplementary Notes } \\
\text { Pascal Vinet, Visiting scientist from Laboratoire de Technologie des Surfaces, } \\
\text { Ecole Centrale de Lyon, B.P. 163, 69131 Ecully Cedex, France; John Ferrante, NASA } \\
\text { Lewis Research Center; John R. Smith, General Motors Research Laboratories, } \\
\text { Warren, Michigan 48090; James H. Rose, Ames Laboratory - USDOE, Iowa State } \\
\text { University, Ames, Iowa 50011. }\end{array}$} \\
\hline \multicolumn{3}{|c|}{ 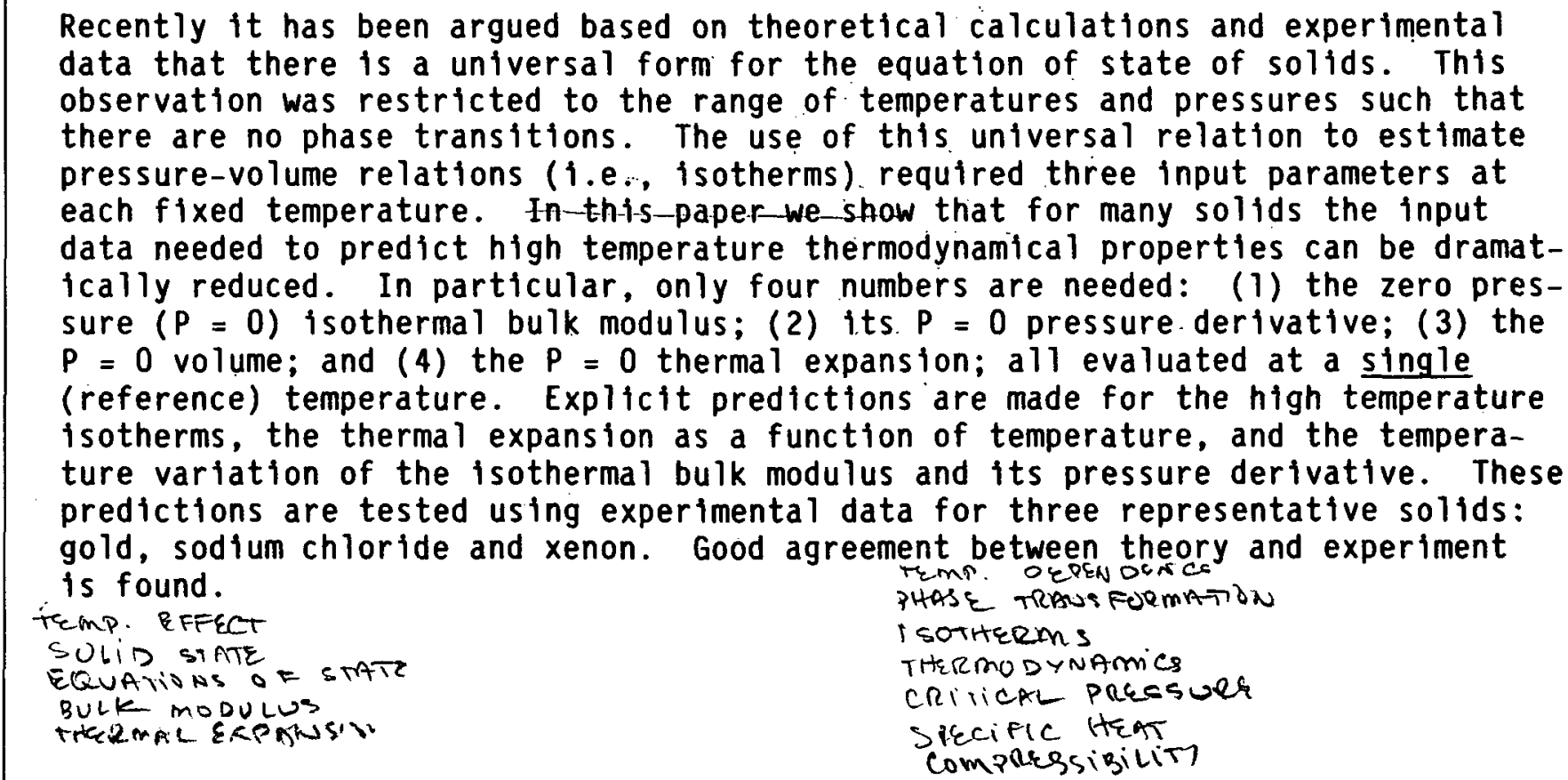 } \\
\hline \multicolumn{3}{|l|}{ 17. Key. Words (Suggested by Author(s)) } \\
\hline $\begin{array}{l}\text { Equation of state } \\
\text { Bulk modulus } \\
\text { Thermal expansion }\end{array}$ & & $\begin{array}{l}\text { ed - unlimited } \\
\text { ory } 77\end{array}$ \\
\hline $\begin{array}{r}\text { 19. Security Classif. (of this report) } \\
\text { Unc lass if ied }\end{array}$ & $\begin{array}{l}\text { Security Classif. (of this page) } \\
\text { Unc las S If ied }\end{array}$ & 21. No. of pages \\
\hline
\end{tabular}

